\title{
Les sociétés anonymes à vocation hôtelière de l'arc lémanique (1826-1914)
}

Julie Lapointe

\section{(2) OpenEdition}

1 Journals

Édition électronique

URL : http://journals.openedition.org/edl/241

DOI : $10.4000 /$ edl. 241

ISSN : 2296-5084

Éditeur

Université de Lausanne

\section{Édition imprimée}

Date de publication : 15 décembre 2010

Pagination : 211-240

ISBN : 978-2-940331-24-6

ISSN : 0014-2026

\section{Référence électronique}

Julie Lapointe, "Les sociétés anonymes à vocation hôtelière de l'arc lémanique (1826-1914) », Études de lettres [En ligne], 4 | 2010, mis en ligne le 15 décembre 2013, consulté le 19 décembre 2020. URL: http://journals.openedition.org/edl/241 ; DOI : https://doi.org/10.4000/edl.241 


\section{LES SOCIÉTÉS ANONYMES À VOCATION HÔTELIÈRE DE L'ARC LÉMANIQUE (1826-1914)}

Partie intégrante du développement touristique de l'Arc lémanique, l'industrie hôtelière a largement contribué à affermir la réussite économique de cette région de la Suisse au XIX ${ }^{\mathrm{e}}$ et au début du XX $\mathrm{XX}^{\mathrm{e}}$ siècle. Créées pour financer la construction des palaces et grands hôtels de l'époque, les sociétés anonymes à vocation hôtelière ont représenté un secteur d'activité profitable qui a su réunir les acteurs de différents milieux: touristique, bancaire, politique, industriel ou commercial. Travaillant en étroite collaboration avec certains architectes, ces sociétés sont devenues de précieux clients pour ces derniers. Depuis la formation de la Société des Bergues de Genève en 1826 jusqu'à celle de la Société du Grand Hôtel de Clarens en 1914, près d'une cinquantaine de sociétés différentes vont voir le jour autour du bassin lémanique. Quel portrait peut-on dresser de ces sociétés et quelle a été leur influence sur l'industrie hôtelière lémanique?

Dans le cadre de la thèse de doctorat que je prépare sur le développement hôtelier de l'Arc lémanique entre le milieu du XIXe siècle et $1914^{1}$, et plus particulièrement sur l'importance de la maîtrise d'un savoir-faire technique dans la réussite de l'offre hôtelière de cette région touristique, je me suis intéressée aux acteurs à l'origine de la construction des grands hôtels et palaces. Bien que certains personnages se soient illustrés de manière marquante dans l'essor de l'industrie hôtelière du Léman à cette époque et figurent parmi les pionniers de l'hôtellerie suisse ${ }^{2}$, il s'avère

I. Sous la direction de Cédric Humair, Maître d'enseignement et de recherche, Section d'histoire, Université de Lausanne et la co-direction du Prof. Laurent Tissot, Institut d'histoire, Université de Neuchâtel.

2. A titre d'exemples, Adolphe-Rodolphe Armleder (1847-1930), Charles-Albert Mayer (1862-1941), Hermann Spahlinger (1852-1934), Ami Chessex (1840-1917) ou encore Alexandre Emery (1850-1931). 
que l'histoire de nombreux établissements est aussi à relier à la création d'entités sociales plus grandes: les sociétés anonymes ou immobilières à vocation hôtelière. Rarement considérées dans la littérature traitant de l'hébergement hôtelier, ces sociétés ont pourtant servi à financer la construction de prestigieux établissements, tels le Beau-Rivage d'Ouchy et le Montreux Palace, ou ont permis l'agrandissement et la transformation d'hôtels plus anciens, à l'instar de l'Hôtel Métropole et de l'Hôtel des Bergues de Genève ou encore de l'ancien Grand Hôtel de Vevey. Mais que savons-nous de ces sociétés? Hormis quelques études bien isolées, la plupart de ces sociétés, près d'une cinquantaine pour l'Arc lémanique (cf. annexe I), nous sont restées pratiquement inconnues jusqu'à aujourd'hui. L'intérêt de cette présente contribution consiste à dégager un portrait plus précis de ces différentes sociétés, d'en montrer leurs buts et d'identifier les principaux acteurs figurant parmi leurs instances dirigeantes. En outre, cette démarche permettra de mettre en évidence le rôle qu'ont joué ces sociétés dans la croissance et l'affermissement de l'industrie hôtelière lémanique à cette époque et nous amènera à discuter du lien étroit qu'elles ont entretenu avec les architectes spécialisés dans la construction hôtelière, en tant que "clients» de ces derniers.

\section{Importance financière de l'industrie hôtelière}

Jusqu'à l'arrivée du premier conflit mondial, l'industrie hôtelière ne cesse de prendre une place toujours plus grande dans l'économie de la Suisse, tout spécialement dans certaines régions peu industrialisées, dont l'Arc lémanique. Souvent relevée dans la littérature traitant de l'histoire de l'hôtellerie, la période qui correspond à celle de la Belle Epoque connaît un développement sans précédent par rapport au nombre et à la taille des établissements construits et destinés à accueillir les étrangers en séjour dans le pays. A Lausanne, on enregistre près de 25 nouveaux hôtels de $1^{\text {er }}$ et $2^{\text {e }}$ rang entre 1900 et $1910^{3}$. Quant à Montreux et ses hauts, le nombre de lits est multiplié par trois entre 1890 et 1912 et les nouveaux hôtels construits entre 1900 et 1912 contiennent une capacité moyenne de 196,5 lits comparativement à 36,5 lits en $1860^{4}$. Estimée à plus d'un

3. L. Tissot, "Hôtels, pensions, pensionnats et cliniques», tableau 1.

4. P. Dupont et S. Frey, "Un paradis encadré», p. 34 sqq. 
milliard, la somme des capitaux investis pour l'année 1912 dans le seul secteur de l'hôtellerie à travers le pays s'avère presque équivalente à celle qui est injectée, à la même époque, dans la compagnie des $\mathrm{CFF}^{5}$. Si l'on compare les années comprises entre 1880 et 1912, les capitaux versés dans l'hôtellerie augmentent de 817 millions de francs, passant de 319 millions à 1136 millions, soit une croissance de 25,5 millions en moyenne chaque année. En divisant ce montant par le nombre d'hôtels en Suisse en 1912, on peut prétendre que l'investissement moyen par hôtel équivalait à environ 317000 francs $^{6}$. Aujourd'hui, cette somme atteindrait plus de 41 millions de francs ${ }^{7}$. Même s'il nous est difficile d'évaluer la répartition exacte de cet investissement dans la région lémanique, on peut toutefois l'estimer à près de $10 \%$ voire $15 \%$ pour $1912^{8}$. Sachant que la part des capitaux étrangers au sein de l'industrie hôtelière est généralement admise comme étant très minime, on ne peut que s'interroger sur l'origine des bailleurs de fonds susceptibles d'avoir participé au rayonnement de cette branche du domaine touristique, d'où l'intérêt d'étudier d'une manière plus approfondie les sociétés anonymes à vocation hôtelière créées à cette époque. En outre, lorsqu'on considère que certaines sociétés, comme celle qui exploite le Grand Hôtel et l'Hôtel des Alpes de Territet ou encore celle du Beau-Rivage à Ouchy, versent respectivement des dividendes allant jusqu'à $12 \%$ et $30 \%$ durant les années précédant 1914, ou que l'action de la société de l'ancien Hôtel Gibbon à Lausanne passe d'une valeur nominale de 500 fr. à 1155 fr. entre 1902 et 1913, il est fort à propos de reconsidérer le rôle joué par l'ensemble de ces sociétés dans la réussite de l'activité touristique de l'Arc lémanique 9 .

5. H.-G. Senn, La Suisse et le tourisme, p. 138.

6. Zentralbureau des Schweizer Hotelier-Vereins (Hrsg.), Zur Erinnerung an die schweizerische Landesaustellung, tableaux $\mathrm{n}^{\text {os }} 14$ et 20. Ce montant correspond à la division de 1135915000 francs par 3585 hôtels répertoriés en 1912, soit 316852 francs.

7. En prenant le PIB de 1912 (4 117 millions de francs) et celui de 2008 (541 827 millions de francs), on arrive environ à cette somme.

8. Zentralbureau des Schweizer Hotelier-Vereins (Hrsg.), Zur Erinnerung an die schweizerische Landesaustellung, tableau $\mathrm{n}^{\circ} 13$. En 1912, le nombre de lits pour les cantons de Genève et de Vaud réunis est de 25070 et celui de la Suisse, 168625. Il faut évidemment retrancher un certain nombre de lits du nombre évalué pour le canton de Vaud, car situés en-dehors de l'Arc lémanique.

9. R. Monneron, Les valeurs de la Bourse de Lausanne, p. 86 sqq. 


\section{Les premières sociétés de l'hôtellerie lémanique}

Bien que la constitution de sociétés anonymes dans l'hôtellerie ne semble se généraliser en Suisse qu'à la fin du XIX ${ }^{\mathrm{e}}$ siècle, on en retrace toutefois un certain nombre avant les années $1880^{10}$. En effet, la plupart des villes lémaniques et du Chablais vaudois vont très tôt assister à la création de sociétés dites à vocation hôtelière, souvent reliée à l'apparition des premiers grands hôtels de la région. Sans trop d'étonnement, les premières sociétés créées dans l'Arc lémanique sont recensées à Genève - Société des Bergues (1826), Société de l'Hôtel de l'Ecu de Genève (1839) et Société de l'Hôtel de la Métropole (1854) - et sont à l'origine de la construction de trois des plus prestigieux établissements de cette ville. A Lausanne, c'est sur les bords du lac que prend forme la première société destinée à l'exploitation d'un hôtel, la Société immobilière d'Ouchy (1857), suivie de la Société de l'Hôtel Suisse, fondée en 1873. Sur la riviera lémanique, la ville de Vevey connaît également ce phénomène puisqu'en 1865, une société immobilière est appelée à financer l'achat de terrains et la construction du Grand Hôtel de Vevey, inauguré deux années plus tard. Enfin, Chexbres accueille une société anonyme en 1864 dont le but est de soutenir l'édification de l'Hôtel du Signal; Bex est l'hôte d'une société immobilière en 1865 et Lavey-les-Bains inscrit une première société anonyme en 1874 . En comparaison avec la partie française du lac Léman, où certaines villes de villégiature, notamment Evian et Thonon, connaissent une affluence grandissante d'étrangers à la même époque, la gestion des grands hôtels lémaniques suisses semble rapidement soumise à la création de ces sociétés anonymes par actions. Jusque dans les années 1840, il est très rare de rencontrer ce genre de structures en Savoie et l'une des premières sociétés créées pour gérer l'exploitation des eaux à Evian, capital touristique de la ville, est d'ailleurs formée par un groupe de propriétaires genevois, en $1844^{11}$.

Io. La prochaine partie est basée sur les onglets des actes de constitution des sociétés anonymes conservés aux Archives cantonales vaudoises (ACV) et aux Archives d'Etat de Genève (AEG). En outre, la Feuille officielle suisse du commerce (FOSC), consultée aux archives de l'Office cantonal du registre du commerce à Moudon, a également servi à cette recherche.

II. F. Breuillaud-Sottas, La prodigieuse ascension des eaux d'Evian (1790-1914), p. 87. Cette société, en activité jusqu'en 1856, est appelée Société des Eaux Minérales d'Evian. Son siège social est à Genève et son capital social est de 100000 francs français. 


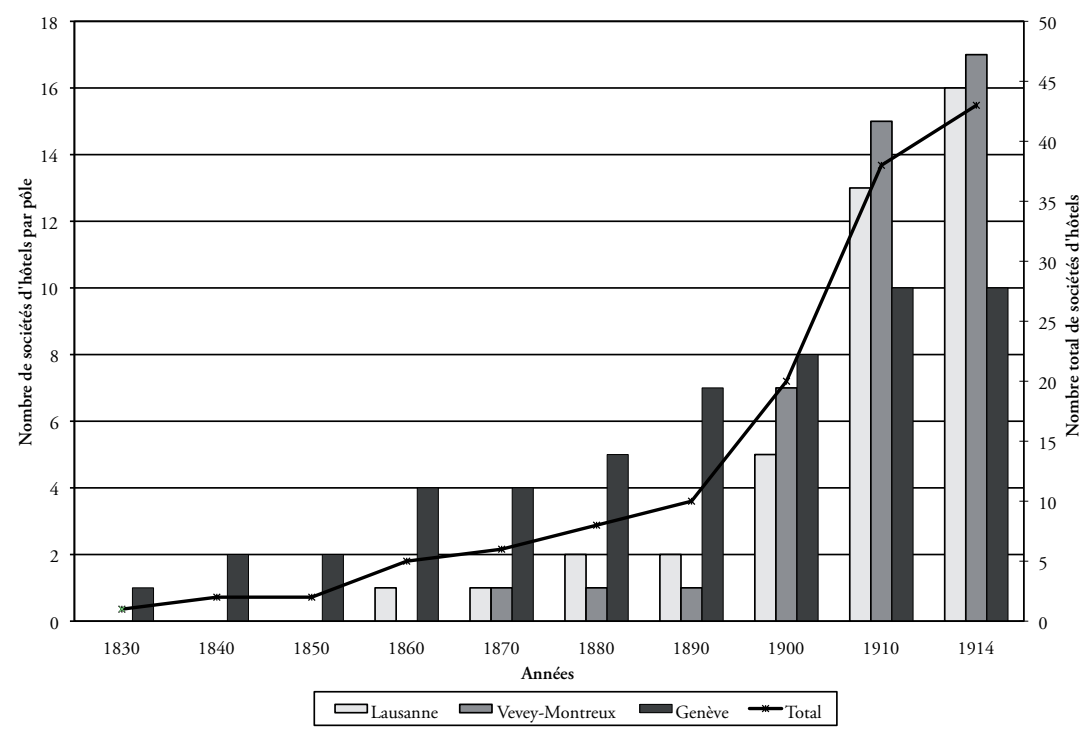

Fig. 1 - Evolution du nombre de sociétés d'hôtels répertoriées dans l'Arc lémanique entre les années 1830 et 1914.

A partir du milieu des années 1890, au moment où l'amélioration de la conjoncture suisse permet une importante reprise de la construction hôtelière, on assiste à une notable augmentation du nombre de nouvelles sociétés créées à l'intérieur du bassin lémanique, et ceci en incluant la rive française du lac. La majorité des grands hôtels et palaces édifiés durant cette période sont presque systématiquement la propriété d'un groupe d'actionnaires et se retrouvent gérés par un conseil d'administration ou un comité de direction. C'est principalement à Lausanne et à Montreux, où l'ouverture de la ligne du Simplon (1906) y est pour beaucoup, que l'on enregistre la plus large part de ces nouvelles sociétés (fig. 1). A Lausanne, l'établissement des hôtels Royal (1909), de la Paix (1910) et Savoy (1911), ainsi que celui du Lausanne-Palace (1915), tous de $1^{\text {re }}$ catégorie, donne naissance à quatre nouvelles sociétés. Dans la région du Haut-Lac, la création du Montreux-Palace (1906), supportée par la Société des Hôtels National \& Cygne (1895), ainsi que celle des grands hôtels de Caux, des Avants, de Chamby, de Clarens et du Mont-Pèlerin, qui relèvent tous d'une société anonyme, atteste encore une fois de ce 
phénomène ${ }^{12}$. Cette pratique ne caractérise toutefois pas la seule région de l'Arc lémanique, mais se rencontre également à Gstaad, à Zermatt ou encore à Berne, pour ne citer que quelques exemples ${ }^{13}$. Très peu d'établissements prestigieux, construits avant la Belle Epoque, vont d'ailleurs demeurer entre les mains d'anciennes familles d'hôteliers, à l'instar de l'Hôtel Beau-Rivage à Genève, toujours sous la direction de la famille Mayer ou encore de l'Hôtel Richemond, longtemps resté sous l'égide des Armleder. Pour illustrer ce fait, l'Hôtel Byron à Villeneuve, l'Hôtel des Alpes à Territet, les hôtels Gibbon et Richemont à Lausanne, l'Hôtel du Lac à Genève, l'Hôtel des Trois Couronnes à Vevey et les hôtels National et du Cygne à Montreux vont tous être rachetés et exploités par des sociétés anonymes à partir des années 1890. Du côté d'Evian, trois des plus grands édifices destinés à l'accueil des étrangers, l'hôtel Splendide (1897), ancien Grand-Hôtel des Bains, l'Hôtel Royal (1909) et l'Hôtel Ermitage (1909), vont aussi devenir la propriété de sociétés, soit celle de la Société des Hôtels (1906) et de la Société de l'Ermitage (1907), deux des filiales de la Société anonyme des Eaux minérales d'Evian créée en $1868^{14}$.

\section{Pourquoi créer des sociétés anonymes à vocation hôtelière?}

Lorsqu'on analyse les statuts des sociétés anonymes pour la période qui se situe entre 1826 et 1914 , on s'aperçoit que la nature de l'objet diffère sensiblement d'une société à l'autre. Si l'on retrouve assez souvent les indications "achat d'hôtel» et "exploitation d'hôtel» parmi les buts visés par ces sociétés, il s'avère intéressant de montrer que certaines d'entre elles se sont constituées pour répondre à des ambitions plus grandes. En effet, plusieurs sociétés créées dans les années 1890 et au début du

I2. La Société immobilière de Caux (1899) est propriétaire du Grand Hôtel de Caux (1893) et du Caux-Palace (1902). On signale encore la Société du Grand Hôtel des Avants (1910), la Société du Grand Hôtel des Narcisses et Buffet Terminus de Chamby sur Montreux (1905) et la Société du Grand Hôtel de Clarens (1914). Pour le MontPèlerin, voir D. Lüthi, «Lavaux-Palace», p. 185 sqq.

I3. Respectivement, la Société du Royal et Winter Palace (1911), la Société des Hôtels Seiler (1908) et la Société anonyme du Bellevue-Palace (1911).

I4. F. Breuillaud-Sottas, La prodigieuse ascension des eaux d'Evian (1790-1914), tableau $\mathrm{n}^{\circ} \mathrm{I}, \mathrm{p} .150$ des annexes. 
$\mathrm{XX}^{\mathrm{e}}$ siècle ont souvent permis de financer des travaux de transformation, d'agrandissement ou de reconstruction d'hôtels qui se seraient avérés difficiles à supporter par un seul propriétaire. L'exemple de la Société de l'Hôtel Byron nous le montre bien. Inauguré à la fin des années 1830, l'Hôtel Byron, alors le plus grand bâtiment hôtelier de la riviera lémanique, passe entre les mains de plusieurs hôteliers et directeurs avant de devenir la propriété d'une société anonyme en janvier $1898^{15}$. Cette société, au capital social de 400000 fr., va parvenir à donner un second souffle à l'établissement en le modernisant de fond en comble. Selon les plans des architectes Georges Rouge (1834-1920) à Lausanne et Jules Clerc (1844-1909) à Clarens, le Byron sera entièrement refait entre 1898 et 1899 et muni des dernières nouveautés techniques, dont un chauffage central à vapeur d'eau et un ascenseur ${ }^{16}$. La publicité que diffuse l'hôtel entre 1897 et 1899 ne manque pas de nous signaler les réalisations accomplies par la société en matière de confort:

Hôtel Byron, Villeneuve. A 5 minutes de Chillon. A 10 minutes de Montreux. Tramway. Position unique. Grand parc ombragé. Recommandé aux familles pour séjour d'été et automne. Cure de lait et de raisins. Gare et bateaux à Villeneuve. Omnibus. H. Gehrig, proprio ${ }^{17}$.

Hôtel Byron, Villeneuve. Ouvert toute l'année. Maison de $1^{\mathrm{er}}$ ordre. Chauffage central à eau et lumière électrique partout. Dernier confort. Ascenseur ${ }^{18}$.

Tout comme cet exemple, d'autres sociétés anonymes se lancent également dans d'importants travaux d'amélioration d'hôtels voire même de construction de nouveaux bâtiments destinés à l'accueil et à la distraction des étrangers. Lorsque la Société du Grand Hôtel des Avants voit

I5. L'hôtel Byron semble avoir appartenu à Gustave Wolff, également propriétaire de l'Hôtel de l'Ecu à Genève, vers 1868, à la famille Jean-Jacques de la Rottaz dans les années 1880 et 1890, ainsi qu'à Hermann Gehrig entre 1897 et 1898 avant d'être racheté par une société anonyme.

I6. R. Flückiger-Seiler, Hotelträume, p. 107 et "Canton de Vaud», Gazette de Lausanne, 19 juillet 1899, p. 3.

17. Publicité de l'hôtel Byron, Journal de Genève, 24 août 1897, p. 3.

I8. Publicité de l'hôtel Byron, Gazette de Lausanne, $1^{\text {er }}$ septembre 1899, p. 4. 
le jour en octobre $1910^{19}$, elle inscrit dans ses statuts qu'en plus d'avoir pour but l'achat et l'exploitation du Grand Hôtel des Avants et de ses dépendances, elle pourrait s'intéresser à acquérir ou à édifier d'autres hôtels, terrains et constructions, soit toutes entreprises de nature à contribuer à son développement. Dans les faits, durant l'année 1911, elle mandate l'architecte Louis Villard (1856-1937) pour l'agrandissement du Grand Hôtel aux Avants, ainsi que pour l'élaboration des plans d'un nouvel hôtel à Sonloup, station supérieure du funiculaire Les AvantsSonloup, qui sera inauguré en 1912. A cette même période, Louis Villard est également le concepteur du Buffet de la Gare et bazar des Avants - vaste chalet avec jeu de quilles et billard - et d'un immeuble locatif et commercial destiné à abriter des boutiques et le bureau de poste, ainsi qu'à loger des étrangers de passage, deux commandes passées par la Société du Grand Hôtel. Cette démarche répond bien au besoin de "garnir» la station des Avants d'infrastructures d'hébergement, de transport et de divertissement afin de concurrencer d'autres stations de villégiature qui se développent simultanément dans la région. Cependant, l'ensemble de ces initiatives n'aurait certainement pas été possible sans le soutien financier d'une société dont la valeur totale des propriétés se chiffre à 4350000 francs.

La Société immobilière de Caux, constituée en février 1899, représente également un cas d'étude intéressant. Indiquant dans ses statuts d'origine que ses activités consisteront à acheter des terrains et à créer et exploiter des hôtels, villas et chalets à Caux, cette société sera toutefois amenée à réaliser un nombre bien plus important de projets qui vont permettre de faire rayonner la station de Caux durant les dernières années de la Belle Epoque. En effet, en plus d'agrandir le Grand Hôtel déjà existant et d'ériger le Palace-Hôtel de Caux, la société fera entre autres construire une chapelle anglaise (1906) ainsi qu'une chapelle catholique (1908), aménagera une piste de bobsleigh (1910), créera une patinoire spécialement destinée au curling (1911), un stand de tir de chasse (1905), aménagera un bureau de poste et télégraphe (1903), s'occupera conjointement avec la commune des Planches de la mise sur pied d'un réseau

I9. En 1910, son conseil d'administration est composé des membres suivants: Alexandre Emery (président), Marc Morel, Armand Piguet, René de Gautard, Jean Russwyl, Franz Spickner, Léon Perret, Louis Dufour père et Louis Rosset. Fritz Rolli est secrétaire mais ne semble pas faire partie du conseil. 
d'égouts (1901) et louera même des locaux à la Municipalité pour l'installation d'une classe d'école primaire (1909) ${ }^{20}$. Par l'étendue de ses activités, la Société immobilière de Caux se rapproche d'une société de développement ou d'utilité publique car en plus de gérer des hôtels, elle se charge de l'épanouissement touristique de la station en optant pour une diversification des services et activités proposés.

D'autres sociétés anonymes s'investissent également dans l'aménagement d'infrastructures aux alentours de leurs hôtels. En 1865, la Société immobilière de l'Hôtel de Vevey a pour principal objet de construire et d'exploiter un hôtel de premier ordre à Vevey et d'acquérir les terrains nécessaires à cet effet, mais elle désire également participer à la création d'une route ou promenade publique qui relierait directement la grande route de Fribourg avec la place publique de Vevey dite du Marché. Même si aucune source ne peut nous confirmer que cette société a réellement contribué à construire cette route, elle s'est cependant engagée dans la réalisation d'une place de débarquement pour les voyageurs devant son établissement, dit le "Débarcadère de Plan", inauguré la même année que le Grand Hôtel, soit en $1868^{21}$. Cette implication d'une société d'intérêt privé dans l'aménagement de commodités publiques rappelle celle de la Société immobilière d'Ouchy qui, en plus d'envisager de construire et d'exploiter un hôtel de premier ordre à Ouchy, avait comme but, en 1857, de créer à ce même endroit un quai et une promenade publique, ainsi que de s'occuper de l'arrangement du port. La société se lancera tout d'abord dans la réfection des quais, mais enchaînera très rapidement et simultanément avec la construction du futur Beau-Rivage d'Ouchy ${ }^{22}$. A Genève, l'exemple de la Société des Bergues reflète également cette volonté d'embellir des espaces riverains dans le but d'y voir se construire des hôtels pour étrangers. Dans ses statuts de 1826, la société n'émet cependant pas encore le désir de procéder à la construction d'un hôtel mais s'avère plutôt impliquée dans le rachat de la Fabrique des Bergues,

20. J. Lapointe, Essor et déclin de la Société immobilière de Caux (1898-1930), p. 33 sqq., et plus généralement, Archives communales de Montreux (AM), PP 152, Société immobilière de Caux, A: Registres de procès-verbaux.

2I. La Société immobilière de l'Hôtel de Vevey participe à raison de $50 \%$ des frais engagés de concert avec la commune de Corsier.

22. L'étude approfondie des activités de cette société fait partie intégrante de mes recherches doctorales en cours et s'effectue à partir des archives privées du Beau-Rivage Palace à Ouchy. 
la construction de maisons et de bâtiments, ainsi que dans l'établissement d'un pont reliant la rive droite du Rhône jusqu'à la Fusterie de la rive gauche. Les plans du premier grand hôtel de Suisse, l'Hôtel des Bergues, sont néanmoins mis au concours trois années plus tard et viennent ajouter une dimension hôtelière aux activités de la société.

\section{Des sociétés créées pour gérer plusieurs hôtels}

Comme mentionné auparavant, certains exemples nous démontrent que l'objectif à l'origine de la création de nombreuses sociétés anonymes dépasse sensiblement la seule vocation hôtelière. Toutefois, d'autres sociétés semblent plutôt avoir été créées pour faciliter la gestion de plusieurs hôtels. Parmi celles-ci figure la Société de l'industrie des hôtels, fondée à Genève, en 1896. Cette société constitue un cas de figure particulièrement intéressant puisqu'elle représente la première initiative lémanique visant à centraliser l'exploitation d'établissements hôteliers de $1^{\text {re }}$ catégorie. Présidée par Henri Galopin (1839-1915) ${ }^{23}$, cette société devient propriétaire des hôtels National, Métropole, du Lac et de l'Hôtel des Bergues de Genève, ainsi que du vaste domaine de Plonjon, alors propriété de la fille du défunt Louis Favre (1826-1879), ingénieur du tunnel du Gothard, qu'elle transformera en un espace de nature, le Parc des Eaux-Vives, où «les étrangers et la population genevoise pourront trouver en plein air [...] de saines distractions ${ }^{24}$. Avec un capital social de 5 millions de francs, dont 2,6 millions en capital-actions, elle constitue la société lémanique la plus fortement capitalisée d'avant la Première Guerre mondiale. Parmi ses fondateurs et administrateurs, on retrouve majoritairement des personnalités genevoises - Eugène Richard (18431925), David Goerger (1851-1912), Hermann Spahlinger ${ }^{25}$ et Henri

23. Henri [ou Henry] Galopin est un banquier genevois, membre et président (en 1887) de l'Association des Intérêts du Commerce et de l'Industrie à Genève, député démocrate au Grand Conseil (1903, 1904 et 1907) et membre de l'Union financière de Genève (dès 1905).

24. "Chronique locale. Parc des Eaux-Vives", Journal de Genève, 13 mai 1897, p. 2.

25. Hermann Spahlinger, en plus d'être propriétaire et directeur de l'Hôtel du Lac, est en outre membre fondateur de la Section des hôteliers de Genève (1893), de la Société suisse des maîtres d'hôtels (1882), président de l'Association des Intérêts de Genève (1894-1895) et conseiller municipal radical (1894-1906). 
Galopin - mais également deux importantes figures du développement touristique de Montreux, Alexandre Emery et Georges Masson (18491920). Selon la FOSC, l'objectif de la société serait de promouvoir le «développement et le perfectionnement de l'industrie des hôtels, spécialement en Suisse " ${ }^{26}$. Créée la même année que l'Exposition nationale de Genève et seulement quelque temps après la Section des hôteliers de Genève (1893), appelée dès 1903 à devenir le Syndicat des Hôteliers de Genève, on peut facilement imaginer que cette société revêt un intérêt majeur pour la bonne marche de l'industrie hôtelière genevoise. Sachant que l'Association des intérêts de Genève, ancêtre de l'Office du Tourisme de Genève (1966), se fonde quelques années auparavant (1885) et que son principal objectif est de «travailler au développement du commerce et de l'industrie de la ville de Genève, principalement en ce qui concerne ses rapports avec les visiteurs et étrangers " ${ }^{27}$, et qu'en outre, son comité doit être constitué d'au moins deux représentants parmi les hôteliers de la ville, on comprend mieux les liens qui unissent l'activité touristique et l'économie genevoise de l'époque.

A Lausanne, en 1902, on assiste également à la création d'une société destinée à gérer plusieurs établissements hôteliers de la ville, la Société du Grand Hôtel, réunissant alors les établissements Beau-Site \& Belvédère ainsi que Richemont, respectivement les anciennes propriétés de JeanAdolphe Schmidt et de Sophie Ritter-Wolbold. Présidée par Ferdinand Jomini (1862-1935) ${ }^{28}$, cette société est vue comme le plus important projet d'exploitation hôtelière à Lausanne. En plus d'avoir procédé au rachat de l'Hôtel Beau-Site $\&$ Belvédère pour 1,5 million de francs et de l'Hôtel Richemont pour la somme de 1 million de francs, elle entreprend en 1912 la construction d'un autre bâtiment d'hébergement touristique, l'Hôtel les Palmiers, qui sera inauguré seulement au mois de juin 1915. Au cours de ces années, l'ensemble des activités de cette société dépassera le seul cadre de l'hôtellerie, mais ce dernier domaine restera l'un

26. Archives de l'Office cantonal du registre du commerce à Moudon, FOSC, 7 avril $1896, n^{\circ} 97$, p. 400.

27. J. de Senarclens, N. van Berchem et J. M. Marquis, L'Hôtellerie genevoise, p. 114. Les auteurs mentionnent que l'Association des Intérêts de Genève et le Syndicat des hôteliers de Genève sont étroitement liés dans leur but de promouvoir Genève.

28. Ferdinand Jomini, alors associé de la Banque Charles Masson \& Cie à Lausanne, est également membre du conseil d'administration de la Société des tramways lausannois et de la Société de l'Hôtel Byron à Villeneuve. 
des plus importants. En novembre 1911, on apprend dans la Gazette de Lausanne que l'assemblée des actionnaires de la société a adopté, à l'unanimité, les modifications des statuts proposés par le conseil d'administration ${ }^{29}$ comprenant entre autres l'élévation du capital social de 1 million à 2,2 millions de francs, ainsi que la transformation de la raison sociale, figurant désormais sous le titre de "Lausanne-Palace» ${ }^{30}$. Or, cette nouvelle appellation sous-entend que cette société financera également la réalisation d'un autre grand hôtel de luxe construit à Lausanne, le Lausanne-Palace, qui ouvre ses portes le 19 juin 1915.

\section{L'architecte dans les sociétés d'hôtels}

Parmi l'ensemble des architectes exerçant leur profession dans le bassin lémanique entre 1830 et 1914, quelques-uns sont devenus de véritables spécialistes de la construction hôtelière. Les noms de Philippe Franel (1796-1867), Ernest Burnat (1833-1922), Charles Nicati (18331884), Louis Henri Maillard (1838-1923), Louis Bezencenet (18431922), Francis Isoz (1856-1910) ou encore Charles-François Bonjour (1870-1961) ne sont de loin pas méconnus et peuvent être rattachés à de nombreux édifices hôteliers construits durant cette période. Souvent présenté comme le cas le plus emblématique de la Belle Epoque, Eugène Jost (1865-1946) reste toutefois l'architecte qui est à l'origine de certains des plus grands et somptueux hôtels construits dans la région, principalement autour de la ville de Montreux. Ayant travaillé aux côtés de deux importants promoteurs hôteliers de l'époque, Alexandre Emery et Ami Chessex, Jost ne compte pas moins d'une quinzaine de projets de construction, transformation, agrandissement ou adjonction qui sont à relier au domaine de l'hôtellerie entre les années 1894 et 1914 . Avec Louis Villard (1856-1937) et Hermann Lavanchy (1865-1913), Eugène Jost figure parmi les architectes les plus prolifiques de Montreux d'avant la Première Guerre mondiale.

29. En 1902, le conseil d'administration de cette société se compose de Ferdinand Jomini (prés.), F. Fiaux (notaire), Mme Ritter-Wolbold, P. Beck-Raccaud, EschenardNeuschwander (de Marseille), Henri Manuel, ainsi que J.-A. Schmidt.

30. "Chronique financière", Gazette de Lausanne, 28 novembre 1911, p. 2. 
En plus d'être les concepteurs des plans des hôtels construits et exploités par les sociétés anonymes, un certain nombre d'architectes ont été intégrés au sein des conseils d'administration ou sont devenus actionnaires de ces mêmes sociétés (cf. annexe II) ${ }^{31}$. A Genève, Joseph Paul Collart (1810-1894), qui fut connu tant par sa carrière d'architecte que par ses divers mandats politiques, est entre autres à l'origine des plans de l'Hôtel de la Métropole (1852-1854), de l'Hôtel de Russie (1855) - hôtel particulier de James Fazy - ainsi que du Grand Hôtel des Bains à Evian (1859-1860). Entre 1868 et 1876, il fait également partie de la Société anonyme de l'Hôtel de la Métropole. Tout comme Collart, l'architecte Bernard-Adolphe Reverdin (1809-1901), connu pour avoir dessiné les plans de l'Hôtel de l'Ecu de Genève en 1839, s'avère faire partie des administrateurs de la Société de l'Ecu de Genève, fondée en décembre de la même année et dont le but initial est de financer la construction de l'hôtel. Il en est membre au moins dès 1858 et jusqu'en 1894. François Durel (1856-1906) semble aussi être l'auteur des plans de l'Hôtel Bellevue, ainsi que l'un des administrateurs de la Société immobilière qui le dirige, dès 1901 et jusqu'en 1906, année de son décès.

A Lausanne, la construction de l'Hôtel de la Paix (1908-1910) revient aux architectes Eugène Monod (1871-1929) et Alphonse Laverrière (1872-1954). Or, Monod est membre du conseil d'administration dès la constitution de la Société du Grand Hôtel de la Paix, en 1908, et le reste au moins jusqu'en 1914. Louis Bezencenet, à qui l'on peut attribuer l'agrandissement de plusieurs grands hôtels lausannois, figure également au sein du conseil de la Société de l'Hôtel Suisse en 1873, ainsi que dans celui de la Société immobilière d'Ouchy, entre 1888 et 1889. Il est en outre actionnaire de cette dernière société. Charles Mauerhofer (18311919) et Adrien van Dorsser (1866-?), associés avec Charles-François Bonjour dans la construction du Royal Hôtel à Lausanne (1907-1909), font respectivement partie du conseil d'administration de la Société de l'Hôtel Royal et de la Société de l'Hôtel Mirabeau. Quant à Bonjour, il est en outre l'un des administrateurs de la Société du Savoy-Hôtel à partir de 1909. A Montreux, Charles Volkart (1862-1918) compte parmi

3I. La suite de cette contribution intègre les premiers résultats de ma thèse de doctorat en cours, réalisée dans le cadre d'un projet de recherche dirigé par Cédric Humair: "Système touristique et culture technique dans l'Arc lémanique: acteurs, réseaux sociaux et synergies (1852-1914)». 
les membres du conseil d'administration de la Société du Grand Hôtel des Narcisses et Buffet Terminus à Chamby sur Montreux, et à Vevey, les architectes Jean Franel (1824-1885) et Marc Franel (1834-1897) sont parmi ceux de la société qui exploite le Grand Hôtel. Charles Volkart et Jean Franel semblent être les concepteurs respectifs des plans des hôtels exploités par lesdites sociétés anonymes.

La présence de ces architectes au sein des conseils d'administration des sociétés reliées à l'hôtellerie lémanique permet d'imaginer que ces derniers ont exercé une certaine influence dans les prises de décisions, comme dans celles qui ont trait à l'introduction de nouveaux équipements, à l'instar des ascenseurs ou de la lumière électrique, destinés à satisfaire les conditions de confort réclamées par la clientèle. Pour demeurer compétitifs et faire face à la concurrence, les grands hôtels de l'époque faisaient constamment l'objet de travaux de modernisation et leurs infrastructures devaient rester à la fine pointe du progrès technique. A cette fin, les dirigeants d'hôtels recouraient aux conseils d'acteurs qualifiés, dont ceux que pouvaient leur prodiguer les architectes. L'étude de fonds hôteliers tend à montrer que les architectes ont joué un rôle non négligeable dans cette course au perfectionnement technique que se livraient alors les établissements hôteliers. A titre d'exemple, en 1911, lorsque l'Hôtel des Trois Couronnes de Vevey décide de transformer son ascenseur hydraulique en un ascenseur électrique, c'est à l'architecte Charles Coigny (1878-1935) qu'elle confie l'installation et la supervision du nouvel appareil. Ce même Charles Coigny était intervenu, quelques années auparavant, dans la transformation des sanitaires de l'hôtel, remplaçant l'unique water-closet mis à la disposition des étrangers au rez-dechaussée de l'hôtel par trois nouveaux "appareils perfectionnés» ${ }^{32}$.

32. Archives communales de Vevey (ACVevey), 95.01, Correspondance d'hôtels, Enveloppe de l'Hôtel des Trois Couronnes, lettre de Charles Coigny au Préfet du district de Vevey datée du 12 janvier 1912 et lettres de Charles Coigny à la Municipalité de la Commune de Vevey datées des 24 et 29 juin 1908. 
Implication des milieux bancaires dans les sociétés anonymes à vocation hôtelière

Dès que le tourisme vaudois prit son essor, malgré les risques que comportait le financement d'hôtels, souvent de grand luxe, plusieurs banques privées jouèrent dans ce secteur un rôle de premier plan. Elles furent ainsi, pour une large part, à l'origine du développement de notre hôtellerie et de nos chemins de fer de montagne ${ }^{33}$.

Cette citation, qui rappelle l'implication des milieux de la finance privée dans le développement de l'industrie touristique de l'Arc lémanique, permet d'imaginer que tout au long du XIX et au début du XXe siècle, des acteurs issus de la sphère bancaire ont pu figurer parmi les promoteurs de l'activité touristique, dont celle qui a trait à la construction d'établissements hôteliers. En effet, plusieurs rentiers ou banquiers privés lausannois - les Bugnion, Bory, Brun, Chavannes, Charrière, Marcel principalement reliés au parti libéral, vont avoir tendance à orienter leurs activités vers 1900 et se spécialiser dans le financement du secteur de la construction et de l'hôtellerie. En outre, le rôle joué par la Banque de Montreux, fondée en 1868, dans l'affirmation de l'industrie touristique de cette région n'est plus à démontrer. L'étude plus approfondie de certaines sociétés de l'hôtellerie, qui révèle la présence de financiers genevois et veveysans parmi les instances dirigeantes, permet également de penser que la réussite de l'ensemble de cette industrie lémanique a été fortement influencée par le soutien d'un tissu bancaire régional. Quelques exemples vont servir à illustrer ce fait.

A Genève, l'un des premiers grands établissements d'accueil à voir le jour est l'Hôtel Métropole, situé sur le Grand Quai en face du Jardin anglais. Edifié selon les plans de l'architecte Joseph Collart, il est, lors de son ouverture officielle à l'été 1857 , complètement éclairé au gaz et muni d'un système d'appareils de sonnerie électrique. Près de quatre ans auront été nécessaires à l'édification de cet hôtel et ses frais de construction sont évalués à 1,2 million de francs, sans compter l'ameublement ${ }^{34}$. L'initiative de cette considérable entreprise hôtelière revient à Christian-Frédéric Kohler (1804-1875), qui crée à cet effet la Société

33. Encyclopédie illustrée du Pays de Vaud, 3, p. 174 sq.

34. "Faits divers", Journal de Genève, 21 août 1857, p. 3. Cet hôtel est sûrement l'un des premiers en Suisse à être complètement éclairé au gaz. 
de l'Hôtel Métropole en mars 1854. Banquier genevois, Kohler est également impliqué dans la création de la ligne de chemin de fer ParisLyon-Méditerranée, administrateur des premières sociétés privées fournisseurs de gaz à Genève et de la Banque de Genève, ainsi que l'un des membres de la Société des agents de change réunis, ancêtre de la Bourse de Genève. Le fait que Kohler provienne du milieu bancaire genevois n'est pas anodin car nous retrouvons d'autres noms de la finance genevoise parmi les administrateurs ou actionnaires de la Société de l'Hôtel Métropole: Jean-Louis Jérome dit John Jérome (?-1901) est un agent de change à Genève et membre du Comptoir d'Escompte de Genève, Charles Galland (1816-1901), agent de change et administrateur de la Compagnie générale de navigation et Hugo Brodhag, banquier et consul d'Allemagne à Genève. En plus des personnages issus du milieu bancaire genevois, on retrouve parmi les autres administrateurs, une importante famille de fabricants de bijouterie et d'horlogerie genevoise, les Rossel, un hôtelier, Alexandre Rufenacht, ainsi que l'architecte de l'hôtel, Joseph Collart.

A Vevey, le rôle des banquiers privés dans le développement d'infrastructures hôtelières et touristiques n'est pas non plus à négliger. La toute première société à vocation hôtelière créée à Vevey au début de 1865, la Société immobilière de l'Hôtel de Vevey, qui est à l'origine de l'édification de l'ancien Grand Hôtel de Vevey, compte parmi les cinq membres de son conseil d'administration initial les banquiers Charles Neyroud, à Vevey, Alexandre Ellissen, à Paris, ainsi que Gustave de Morsier, à Genève ${ }^{35}$. Les deux autres membres sont l'architecte de l'hôtel, Jean Franel, et Louis Naville-Todd, propriétaire à Genève. Au début des années 1880, la société est présidée par nul autre que William Cuénod (1841-1920), de la banque Cuénod, W. \& Cie, et ce jusqu'en 1894, quelque temps avant que la société ne revende son hôtel à Eugène Michel, alors directeur de l'établissement. En février 1904, une seconde société, la Société du Grand Hôtel de Vevey et Palace Hôtel, se constitue et procède au rachat de l'hôtel avec ses dépendances et son mobilier. Son président est encore une fois un acteur central des milieux financiers de la ville, Eugène Paschoud (?-1918). Directeur du Crédit du Léman à Vevey, il restera à la tête de cette nouvelle société au moins jusqu'en

35. Annonce de la Société immobilière de l'Hôtel de Vevey, Gazette de Lausanne, 22 mars 1865, p. 4. 
1914. Avec un capital social de 456500 fr., «fourni pour la plus grande part par des personnalités veveysannes» ${ }^{36}$, la Société du Grand Hôtel de Vevey va permettre entre autres de financer la modernisation des infrastructures de l'établissement, dont la mise en place d'un chauffage central. Au sein de son conseil d'administration, on retrouve les acteurs suivants: Eugène Paschoud, Henri Schobinger, Emile Romang, Paul Taverney, William Niess fils, hôtelier au Mont-Pèlerin, Charles-Frédéric Butticaz, hôtelier à Lausanne, et Koelher-Mooser, hôtelier à Vevey ${ }^{37}$.

Mis à part ces cas de figure, le nom d'Albert Cuénod (1846-1909) mérite d'être également souligné. Cette personnalité, à la tête de la banque A. Cuénod \& Cie, va considérablement s'impliquer dans l'essor de l'industrie hôtelière de la riviera lémanique. Administrateur de la Société immobilière de Caux, vice-président de la Société de l'Hôtel des Trois Couronnes à Vevey et de la Société du Grand Hôtel et Hôtel des Alpes à Territet, on le retrouve également comme président de la Société du Majestic Palace Hôtel, créée à Vevey en mars 1906, et dont le but est de financer la construction d'un majestueux établissement hôtelier à Nice. Important bailleur de fonds de l'industrie chocolatière veveysanne, il est avec Gabriel Montet (1848-1915) et René de Gautard (1871-1939), l'une des figures veveysannes majeures impliquées à la fois dans l'essor industriel de la ville que dans la sphère touristique ${ }^{38}$.

Parmi les plus importants banquiers privés lausannois, on relève tout d'abord la présence de Ferdinand de Charrière de Sévery (1849-1922) comme administrateur de la Société du Grand Hôtel de la Paix. A l'origine de la création de la banque Charrière $\&$ Roguin en 1882, Charrière de Sévery est aussi l'un des fondateurs et président du conseil d'administration de la Banque d'Escompte et de Dépôts, absorbée plus tard par la Société de Banque Suisse, dont il demeure l'un des administrateurs.

36. Feuille d'Avis de Vevey, $1^{\mathrm{er}}$ mars 1904.

37. "Chronique financière», Gazette de Lausanne, 23 avril 1913, p. 5.

38. Gabriel Montet, banquier privé à Vevey, est administrateur des sociétés Grand Hôtel Monney et Beau-Séjour au Lac, de l'Hôtel du Pont Terminus et Buffet de la Gare à Vevey, ainsi que dans la Société immobilière de l'Hôtel de Vevey. René de Gautard, banquier à St-Légier et à Vevey, fait aussi partie de l'Union foncière Suisse. Il sera impliqué dans plusieurs sociétés d'hôtels de la région dont la Société de l'Hôtel des Trois Couronnes, du Grand Hôtel et Hôtel des Alpes à Territet, la Société immobilière de Caux, la Société du Grand Hôtel des Avants, ainsi que dans la Société du Majestic Palace Hôtel de Nice et des Hôtels Seiler à Zermatt. 
Au sein de la Société du Grand Hôtel de la Paix, il s'implique activement et représente l'un des principaux initiateurs du projet. Sa banque assure d'ailleurs le compte de crédit de construction de l'hôtel, ouvert le $1^{\text {er }}$ août 1910 , dont le coût total s'est élevé à 2,4 millions de francs, soit 800000 fr. de plus que le devis prévu ${ }^{39}$. Hormis l'implication de ce banquier lausannois dans la sphère hôtelière, on remarque également celle de Julien Chavannes (1840-1914), de la Banque Morel, Chavannes, Gunthert \& Cie, à titre de président de la Société de l'Hôtel suisse. Cette société, qui fut impliquée dans la construction d'un hôtel situé à proximité de la gare de Lausanne au début des années 1870, comptait en outre les banquiers lausannois Charles Masson (1864-1939), Henri Pittet, Henri Siber (1831-1905) et A. L. Dutoit parmi les membres de son conseil d'administration. Suite à d'importants glissements de terrain survenus durant les premiers mois de construction de l'hôtel, soit à l'été 1874, la société se voit dans l'obligation de démolir le bâtiment en cours et de procéder à la liquidation de ses actifs. Enfin, à Villeneuve, la Société de l'Hôtel Byron, constituée en 1893 dans le but de reconstruire l'établissement et de l'exploiter, est administrée entre autres par trois acteurs issus du monde de la finance: Ferdinand Jomini, Alphonse Vallotton (?-1903), banquier à Lausanne et Jean Russwyl, fondé de pouvoirs puis directeur de la Banque de Montreux.

\section{Conclusion}

Souvent constituées pour permettre d'amasser les sommes nécessaires à la construction, l'agrandissement ou la rénovation d'un ou de plusieurs hôtels, les sociétés à vocation hôtelière ont non seulement permis d'accroître et d'améliorer l'offre d'hébergement au sein des principales villes du Léman, mais elles ont également joué un rôle essentiel dans la croissance et l'affermissement de certains secteurs de l'activité économique locale et régionale. Cette contribution, qui a montré les liens entre les architectes, les banquiers et les promoteurs de l'industrie hôtelière lémanique, aura servi à donner un premier aperçu des réseaux sociaux

39. "Lausanne. La transformation de Lausanne", Gazette de Lausanne, 12 février 1908, p. 3 ; "Lausanne. Nouveau Lausanne», Gazette de Lausanne, 19 mars 1908, p. 3 et "L'Hôtel de la paix», Bulletin technique de la Suisse romande, 37 (1911), p. 168. 
Fig. 2 - En-tête d'une enveloppe représentant les hôtels Beau-Site et Riche-Mont à Lausanne, propriétés de la Société Anonyme Le Grand Hôtel (1904) (MHL).

susceptibles d'avoir influencé la réussite de cette branche du tourisme durant la période précédant l'arrivée du premier conflit mondial. Il n'en reste pas moins que l'étude des sociétés reliées à l'hôtellerie s'avère bien souvent complexe, puisqu'à défaut de pouvoir systématiquement recourir à des fonds d'archives privés, la recherche doit reposer sur l'analyse d'autres types de documents, à l'instar des journaux ou des actes notariés. Et dans ce cas, il arrive qu'un simple en-tête de papier, heureusement conservé, puisse se révéler être une source précieuse...

Julie Lapointe doctorante FNS

Section d'histoire, Université de Lausanne 


\section{TABLEAU DES SOCIÉTÉS D'HÔTELS RÉPERTORIÉES DANS L'ARC LÉMANIQUE ENTRE 1826 ET 1914}

Abréviations: LS: Lausanne; VM: Vevey et Montreux; GE: Genève; E: Evian-les-Bains; VI: Villeneuve.

\begin{tabular}{|l|c|c|}
\hline \multicolumn{1}{|c|}{ Nom de la société } & Année & Pôle \\
\hline Société des Bergues & 1826 & GE \\
\hline Société de l'Hôtel de l'Ecu de Genève & 1839 & GE \\
\hline Société de l'Hôtel de la Métropole & 1854 & GE \\
\hline Société des Hôtels garnis de Genève & 1855 & GE \\
\hline Société immobilière d'Ouchy & 1857 & LS \\
\hline Société immobilière de l'Hôtel de Vevey & 1865 & VM \\
\hline Société de l'Hôtel Suisse & 1873 & LS \\
\hline Société immobilière \& industrielle de Chamonix & 1875 & GE \\
\hline Société de l'Hôtel National de Genève & 1881 & GE \\
\hline Société anonyme de Champel Beau-Séjour & 1887 & GE \\
\hline Société du Grand Hôtel (et Hôtel des Alpes) de Territet & 1891 & VM \\
\hline Société Pension Beau-Séjour & 1892 & LS \\
\hline Société de l'Hôtel des Trois Couronnes à Vevey & 1895 & VM \\
\hline Société des Hôtels National \& Cygne à Montreux & 1895 & VM \\
\hline Société de l'Industrie des Hôtels & 1896 & GE \\
\hline Société de l'Hôtel du Pont Terminus et Buffet de la Gare à Vevey & 1896 & VM \\
\hline Société de l'Hôtel du Grand-Pont & 1896 & LS \\
\hline Société de l'Hôtel du Nord à Lausanne & 1898 & LS \\
\hline Société de l'Hôtel Byron & 1898 & VI \\
\hline Société immobilière de Caux & 1899 & VM \\
\hline
\end{tabular}




\begin{tabular}{|l|c|c|}
\hline \multicolumn{1}{|c|}{ Nom de la société } & Année & Pôle \\
\hline Société de l'Hôtel Breuer & 1899 & VM \\
\hline Société de l'Hôtel du Belvédère (au Mont-Pèlerin) & 1900 & VM \\
\hline Société immobilière de l'Hôtel Bellevue & 1901 & GE \\
\hline $\begin{array}{l}\text { Le «Grand Hôtel» (Société anonyme des Hôtels Beau-Site et } \\
\text { Riche-Mont) }\end{array}$ & 1902 & LS \\
\hline Hôtel Gibbon, Société anonyme & 1902 & LS \\
\hline Société du Grand Hôtel du Mont-Pèlerin & 1903 & VM \\
\hline Société Grand Hôtel Monney et Beau Séjour au Lac & 1904 & VM \\
\hline Société du Grand Hôtel de Vevey et Palace Hôtel & 1904 & VM \\
\hline Société anonyme du Pélerin-Palace Hôtel & 1904 & VM \\
\hline Société anonyme du Grand Hôtel Bon Port & 1905 & VM \\
\hline Société de l'Hôtel Moderne & 1905 & GE \\
\hline $\begin{array}{l}\text { Société du Grand Hôtel des Narcisses et Buffet Terminus de } \\
\text { Chamby sur Montreux }\end{array}$ & 1905 & VM \\
\hline Société immobilière de Ben Posta (Hôtel Cécil), Lausanne & 1905 & LS \\
\hline Société anonyme de l'Hôtel Royal & 1906 & LS \\
\hline Société des Hôtels & 1906 & E \\
\hline Société de l'Ermitage & 1907 & E \\
\hline Société du Grand Hôtel de la Paix & 1908 & LS \\
\hline Société anonyme «Savoy-Hôtel» & 1909 & LS \\
\hline Société Immobilière du Signal à Lausanne & 1909 & LS \\
\hline Société du Grand Hôtel des Avants & 1910 & VM \\
\hline Société anonyme de l'Hôtel Terminus & VM \\
\hline $\begin{array}{l}\text { «Lausanne-Palace» anciennement le «Grand Hôtel» SA des } \\
\text { Hôtels Beau-Site et Riche-Mont }\end{array}$ & VM \\
\hline Société anonyme de l'Hôtel Hermitage & LS \\
\hline Société anonyme de l'Hôtel Mirabeau & 1911 & LS \\
\hline Société immobilière de l'Hôtel du Simplon & LS \\
\hline Société du Grand Hôtel de Clarens & 1911 \\
\hline
\end{tabular}




\section{ANNEXE II}

\section{TABLEAU DES ARCHITECTES IMPLIQUÉS DANS LA CONSTRUCTION HÔTELIÈRE LÉMANIQUE ET DANS LES SOCIÉTÉS D'HÔTELS ENTRE 1830 ET 1914}

Abréviations: GE: Genève; LS: Lausanne; MO: Montreux; VE: Vevey;

VI: Villeneuve; E: Evian-les-Bains; Agrand. : Agrandissement;

Exhaus. : Exhaussement; Surélév. : Surélévation; Constr.: Construction;

Transf.: Transformation; Admin. : Administrateur;

Action. : Actionnaire.

\begin{tabular}{|c|c|c|c|}
\hline Nom de l'architecte & Pôle(s) & $\begin{array}{l}\text { Réalisation(s) hôtelière(s) } \\
\text { dans l'Arc lémanique }\end{array}$ & $\begin{array}{l}\text { Administrateur et/ou } \\
\text { actionnaire dans une } \\
\text { (des) société(s) d'hôtel(s) }\end{array}$ \\
\hline $\begin{array}{l}\text { Bertolini, Jean- } \\
\text { Baptiste (1822-1883) }\end{array}$ & LS & $\begin{array}{l}\text { Hôtel Beau-Rivage à } \\
\text { Ouchy (1857-1861) }\end{array}$ & \\
\hline $\begin{array}{l}\text { Bezencenet, Louis } \\
(1843-1922)\end{array}$ & LS & $\begin{array}{l}\text { Agrand. de l'Hôtel } \\
\text { Gibbon (1904); Agrand. } \\
\text { de l'Hôtel Beau-Séjour } \\
\text { (1904); Agrand. de } \\
\text { l'Hôtel Victoria (1906); } \\
\text { Annexe du Beau-Rivage } \\
\text { Palace à Ouchy (1906- } \\
\text { 1908) }\end{array}$ & $\begin{array}{l}\text { Société immobilière } \\
\text { d'Ouchy (admin. et } \\
\text { action.); Société de } \\
\text { l'Hôtel Suisse à LS }\end{array}$ \\
\hline $\begin{array}{l}\text { Bizot, Auguste } \\
(1874-1939)\end{array}$ & LS & & $\begin{array}{l}\text { Société anonyme de } \\
\text { l'Hôtel Royal à LS } \\
\text { (admin. et action.) }\end{array}$ \\
\hline Boët, Louis (?-?) & MO & $\begin{array}{l}\text { Agrand. Hôtel } \\
\text { Bonivard (1904); } \\
\text { Constr. d'une aile à } \\
\text { l'Hôtel de Paris (1906); } \\
\text { Transf. et agrand. Hôtel } \\
\text { du Lac (1912) }\end{array}$ & \\
\hline
\end{tabular}




\begin{tabular}{|c|c|c|c|}
\hline Nom de l'architecte & Pôle(s) & $\begin{array}{l}\text { Réalisation(s) hôtelière(s) } \\
\text { dans l'Arc lémanique }\end{array}$ & $\begin{array}{l}\text { Administrateur et/ou } \\
\text { actionnaire dans une } \\
\text { (des) société(s) d'hôtel(s) }\end{array}$ \\
\hline $\begin{array}{l}\text { Bonjour, Charles- } \\
\text { François } \\
(1870-1961)\end{array}$ & LS & $\begin{array}{l}\text { Hôtel Eden (1905); Hô- } \\
\text { tel Royal (1907-1909); } \\
\text { Hôtel Mirabeau (1910); } \\
\text { transf. de l'Hôtel Beau- } \\
\text { Séjour (1911-1912) }\end{array}$ & $\begin{array}{l}\text { Société anonyme «Savoy- } \\
\text { Hôtel » à LS (admin. et } \\
\text { action.) ; Société } \\
\text { anonyme de l'Hôtel } \\
\text { Royal à LS (action.) }\end{array}$ \\
\hline $\begin{array}{l}\text { Brunnarius, Ernest } \\
(1857-1901)\end{array}$ & E & $\begin{array}{l}\text { Hôtel Splendide (1898) } \\
\text { à Evian-les-Bains }\end{array}$ & \\
\hline $\begin{array}{l}\text { Burnat, Ernest } \\
(1833-1922)\end{array}$ & $\begin{array}{l}\text { VE } \\
\mathrm{MO}\end{array}$ & $\begin{array}{l}\text { Hôtel des Crêtes à } \\
\text { Clarens (1880); Grand } \\
\text { Hôtel du Lac à Vevey } \\
(1866-1868) \text {; Hôtel } \\
\text { National à Montreux } \\
(1872-1874) \text {; Hôtel- } \\
\text { Pension du Châtelard à } \\
\text { Clarens (ouv. en 1874); } \\
\text { Agrand. et transf. de } \\
\text { l'Hôtel-Pension de } \\
\text { Famille (1900) }\end{array}$ & \\
\hline $\begin{array}{l}\text { Chamorel-Garnier, } \\
\text { Charles-François } \\
(1868-1934)\end{array}$ & LS & $\begin{array}{l}\text { Hôtel Beau-Site et } \\
\text { Belvèdre (1897); Hôtel } \\
\text { des Palmiers (1912- } \\
\text { 1913); Lausanne-Palace } \\
(1915)\end{array}$ & \\
\hline $\begin{array}{l}\text { Chessex, Georges } \\
(1868-1932)\end{array}$ & LS & $\begin{array}{l}\text { Hôtel Beau-Site et } \\
\text { Belvèdre (1897); Hôtel } \\
\text { des Palmiers (1912- } \\
\text { 1913); Lausanne-Palace } \\
(1915)\end{array}$ & \\
\hline $\begin{array}{l}\text { Clerc, Jules } \\
(1844-1909)\end{array}$ & $\begin{array}{l}\mathrm{MO} \\
\mathrm{VI}\end{array}$ & $\begin{array}{l}\text { Surélé. Hôtel Mont- } \\
\text { Fleuri (1894); Transf. } \\
\text { Hôtel Byron (1899) }\end{array}$ & \\
\hline $\begin{array}{l}\text { Coigny, Charles } \\
(1878-1935)\end{array}$ & VE & $\begin{array}{l}\text { Hôtel Beau-Séjour à VE } \\
\text { (1907); Transf. à l'Hôtel } \\
\text { des Trois Couronnes à } \\
\text { VE (1912); Hôtel } \\
\text { Touring et Gare à VE }\end{array}$ & \\
\hline
\end{tabular}




\begin{tabular}{|c|c|c|c|}
\hline Nom de l'architecte & Pôle(s) & $\begin{array}{l}\text { Réalisation(s) hôtelière(s) } \\
\text { dans l'Arc lémanique }\end{array}$ & $\begin{array}{l}\text { Administrateur et/ou } \\
\text { actionnaire dans une } \\
\text { (des) société(s) d'hôtel(s) }\end{array}$ \\
\hline $\begin{array}{l}\text { Collart, Joseph } \\
(1810-1894)\end{array}$ & $\begin{array}{c}\mathrm{GE} \\
\mathrm{E}\end{array}$ & $\begin{array}{l}\text { Hôtel Métropole à GE } \\
\text { (1852-1854); Grand } \\
\text { Hôtel des Bains à Evian- } \\
\text { les-Bains; Grand Hôtel } \\
\text { de Russie (1855) }\end{array}$ & $\begin{array}{l}\text { Société de l'Hôtel de la } \\
\text { Métropole à GE }\end{array}$ \\
\hline $\begin{array}{l}\text { Convert, Robert } \\
(1860-1918)\end{array}$ & VE & $\begin{array}{l}\text { Agrand. de l'Hôtel des } \\
\text { Trois Couronnes (1893- } \\
1894)\end{array}$ & \\
\hline $\begin{array}{l}\text { Daulte, Alfred } \\
(1874-1922)\end{array}$ & $\mathrm{MO}$ & $\begin{array}{l}\text { Grand Hôtel de Territet } \\
\text { (1906); Caux-Palace } \\
\text { (1907) }\end{array}$ & \\
\hline $\begin{array}{l}\text { Dessaules, Louis } \\
(1875-1953)\end{array}$ & $\mathrm{MO}$ & Hôtel Meurice (1913) & \\
\hline $\begin{array}{l}\text { Dorsser, Adrien van } \\
(1866-?)\end{array}$ & LS & $\begin{array}{l}\text { Hôtel Royal (1907- } \\
\text { 1909); Hôtel Mirabeau } \\
\text { (1910); Transf. de } \\
\text { l'Hôtel Beau-Séjour } \\
\text { (1911-1912) }\end{array}$ & $\begin{array}{l}\text { Société de l'Hôtel } \\
\text { Mirabeau à LS; Société } \\
\text { anonyme de l'Hôtel } \\
\text { Hermitage à LS; Société } \\
\text { anonyme «Savoy-Hôtel» } \\
\text { à LS (action.); Société } \\
\text { anonyme de } \\
\text { l'Hôtel Royal à LS } \\
\text { (action.); Société } \\
\text { anonyme du Royal et } \\
\text { Winter Palace, Gstaad }\end{array}$ \\
\hline $\begin{array}{l}\text { Durel, François } \\
(1856-1906)\end{array}$ & GE & $\begin{array}{l}\text { Hôtel Bellevue à Genève } \\
(1901-1902)\end{array}$ & $\begin{array}{l}\text { Société immobilière de } \\
\text { l'Hôtel Bellevue à GE }\end{array}$ \\
\hline $\begin{array}{l}\text { Fraisse, Henry } \\
(1804-1841)\end{array}$ & LS & $\begin{array}{l}\text { Hôtel Gibbon (1837- } \\
1839)\end{array}$ & \\
\hline $\begin{array}{l}\text { Franel, Jean } \\
(1824-1885)\end{array}$ & VE & $\begin{array}{l}\text { Grand Hôtel de Vevey } \\
(1865-1867)\end{array}$ & $\begin{array}{l}\text { Société immobilière de } \\
\text { l'Hôtel de Vevey }\end{array}$ \\
\hline $\begin{array}{l}\text { Franel, Marc } \\
(1834-1897)\end{array}$ & VE & & $\begin{array}{l}\text { Société immobilière de } \\
\text { l'Hôtel de Vevey }\end{array}$ \\
\hline
\end{tabular}




\begin{tabular}{|c|c|c|c|}
\hline Nom de l'architecte & Pôle(s) & $\begin{array}{l}\text { Réalisation(s) hôtelière(s) } \\
\text { dans l'Arc lémanique }\end{array}$ & $\begin{array}{l}\text { Administrateur et/ou } \\
\text { actionnaire dans une } \\
\text { (des) société(s) d'hôtel(s) }\end{array}$ \\
\hline $\begin{array}{l}\text { Franel, Philippe } \\
(1796-1867)\end{array}$ & $\begin{array}{l}\text { VE } \\
\mathrm{MO}\end{array}$ & $\begin{array}{l}\text { Hôtel des Trois } \\
\text { Couronnes à Vevey } \\
(1840-1842) ; \text { Hôtel du } \\
\text { Cygne I (1835-1836); } \\
\text { Hôtel du Righi vaudois } \\
\text { à Glion (1854-1855); } \\
\text { Hôtel du Cygne II } \\
\text { (1863-1864); Pension } \\
\text { Chemenin à Vevey } \\
\text { devenue Park-Hôtel } \\
\text { Mooser (1867); Villa } \\
\text { Mirabeau (1841) deve- } \\
\text { nue Hôtel Roy en } 1874\end{array}$ & \\
\hline $\begin{array}{l}\text { Gignoux, Jean-Marie } \\
\text { (?-?) }\end{array}$ & GE & Hôtel de la Paix (1863) & \\
\hline $\begin{array}{l}\text { Goss, Jacques-Elysée } \\
(1839-1921)\end{array}$ & GE & $\begin{array}{l}\text { Hôtel National (1874- } \\
1875)\end{array}$ & \\
\hline $\begin{array}{l}\text { Hebrard, Jean-Albert } \\
\text { (?-?) }\end{array}$ & $\mathrm{E}$ & Hôtel Royal (1909) & \\
\hline $\begin{array}{l}\text { Isoz, Francis } \\
(1856-1910)\end{array}$ & LS & $\begin{array}{l}\text { Hôtel International } \\
\text { devenu Hôtel de } \\
\text { Lausanne (1904); Hôtel } \\
\text { Riche-Mont (1901); } \\
\text { Hôtel du Château } \\
\text { d'Ouchy (1888-1893) }\end{array}$ & \\
\hline $\begin{array}{l}\text { Jost, Eugène } \\
(1865-1946)\end{array}$ & $\begin{array}{l}\mathrm{LS} \\
\mathrm{MO}\end{array}$ & $\begin{array}{l}\text { Transf. Hôtel des Alpes } \\
\text { à Territet (1895); Hôtel } \\
\text { de l'Europe à MO (env. } \\
\text { 1897); Transf. Hôtel } \\
\text { National à MO (1898); } \\
\text { Caux-Palace (1900- } \\
\text { 1902); Hôtel des Alpes } \\
\text { à Territet (1903-904); } \\
\text { Montreux-Palace (1904- } \\
\text { 1906); Annexe du Beau- } \\
\text { Rivage Palace à Ouchy } \\
\text { (1905-1908) }\end{array}$ & $\begin{array}{l}\text { Société des Hôtel } \\
\text { National \& Cygne à } \\
\text { Montreux (action.) }\end{array}$ \\
\hline
\end{tabular}




\begin{tabular}{|c|c|c|c|}
\hline Nom de l'architecte & Pôle(s) & $\begin{array}{l}\text { Réalisation(s) hôtelière(s) } \\
\text { dans l'Arc lémanique }\end{array}$ & $\begin{array}{l}\text { Administrateur et/ou } \\
\text { actionnaire dans une } \\
\text { (des) société(s) d'hôtel(s) }\end{array}$ \\
\hline $\begin{array}{l}\text { Junod, Jean Marc } \\
\text { Louis (1806-1873) }\end{array}$ & $\begin{array}{l}\text { GE } \\
\text { VI }\end{array}$ & $\begin{array}{l}\text { Hôtel de la Couronne à } \\
\text { Genève }(1835) \text {; Hôtel } \\
\text { Byron à Villeneuve } \\
(1837-1840)\end{array}$ & \\
\hline $\begin{array}{l}\text { Krafft, Antony } \\
(1831-1910)\end{array}$ & GE & $\begin{array}{l}\text { Hôtel Beau-Rivage } \\
(1865) \text {; Hôtel } \\
\text { d'Angleterre (1871- } \\
1872)\end{array}$ & \\
\hline $\begin{array}{l}\text { La Harpe, Achille de } \\
(1807-1887)\end{array}$ & LS & $\begin{array}{l}\text { Hôtel Beau-Rivage à } \\
\text { Ouchy (1857-1861) }\end{array}$ & \\
\hline $\begin{array}{l}\text { Lavanchy, Hermann } \\
(1865-1913)\end{array}$ & $\mathrm{MO}$ & $\begin{array}{l}\text { Hôtel-pension } \\
\text { International (1894- } \\
\text { 1896); Hôtel de Londres } \\
(1901) \text {; Surélév. Hôtel } \\
\text { de la Tonhalle (1901); } \\
\text { Agrand. Hôtel de } \\
\text { l'Europe (1905-1907); } \\
\text { Agrand. Hôtel des Alpes } \\
\text { à Glion (1906); Transf. } \\
\text { et agrand. Hôtel Roth } \\
\text { (1908) }\end{array}$ & \\
\hline $\begin{array}{l}\text { Laverrière, Alphonse } \\
(1872-1954)\end{array}$ & LS & $\begin{array}{l}\text { Hôtel de la Paix à } \\
\text { Lausanne (1908-1910) }\end{array}$ & \\
\hline $\begin{array}{l}\text { Maillard, Louis } \\
\text { Henri (1838-1923) }\end{array}$ & $\begin{array}{l}\mathrm{VE} \\
\mathrm{MO}\end{array}$ & $\begin{array}{l}\text { Hôtel Roth à } \\
\text { Clarens (1873-1874); } \\
\text { Grand-Hôtel à Territet } \\
\text { (1887-1888); Hôtel- } \\
\text { Pension de Famille à VE } \\
\text { (1889-1890); Grand } \\
\text { Hôtel de Caux (1890- } \\
\text { 1893); Agrand. du Righi } \\
\text { Vaudois à Glion (1895- } \\
\text { 1896); Agrand. de } \\
\text { l'Hôtel des Trois } \\
\text { Couronnes à VE (1893- } \\
\text { 1894) }\end{array}$ & $\begin{array}{l}\text { SA de l'Auberge de } \\
\text { Famille (membre } \\
\text { fondateur) }\end{array}$ \\
\hline $\begin{array}{l}\text { Mauerhofer, Charles } \\
(1831-1919)\end{array}$ & LS & $\begin{array}{l}\text { Hôtel Royal (1907- } \\
1909)\end{array}$ & $\begin{array}{l}\text { Société anonyme de } \\
\text { l'Hôtel Royal à LS } \\
\text { (admin. et action.) }\end{array}$ \\
\hline
\end{tabular}




\begin{tabular}{|c|c|c|c|}
\hline Nom de l'architecte & Pôle(s) & $\begin{array}{l}\text { Réalisation(s) hôtelière(s) } \\
\text { dans l'Arc lémanique }\end{array}$ & $\begin{array}{l}\text { Administrateur et/ou } \\
\text { actionnaire dans une } \\
\text { (des) société(s) d'hôtel(s) }\end{array}$ \\
\hline $\begin{array}{l}\text { Melley, Charles } \\
(1855-1935)\end{array}$ & LS & Hôtel Cécil (1905) & $\begin{array}{l}\text { Société immobilière de } \\
\text { Ben-Posta (Hôtel Cécil) } \\
\text { à LS }\end{array}$ \\
\hline $\begin{array}{l}\text { Meyer, Henri } \\
(1856-1930)\end{array}$ & $\mathrm{MO}$ & Hôtel des Alpes (1906) & \\
\hline $\begin{array}{l}\text { Monod, Eugène } \\
(1871-1929)\end{array}$ & LS & $\begin{array}{l}\text { Hôtel de la Paix à } \\
\text { Lausanne (1908-1910) }\end{array}$ & $\begin{array}{l}\text { Société du Grand Hôtel } \\
\text { de la Paix à LS }\end{array}$ \\
\hline $\begin{array}{l}\text { Nicati, Charles } \\
(1833-1884)\end{array}$ & $\begin{array}{l}\mathrm{VE} \\
\mathrm{MO}\end{array}$ & $\begin{array}{l}\text { Grand Hôtel du Lac à } \\
\text { Vevey (1866-1868); } \\
\text { Hôtel National (1873- } \\
\text { 1874); Agrand. et transf. } \\
\text { de l'Hôtel-Pension de } \\
\text { Famille (1900) }\end{array}$ & \\
\hline $\begin{array}{l}\text { Olivet, Alfred } \\
(1863-1942)\end{array}$ & GE & $\begin{array}{l}\text { Transf. Hôtel } \\
\text { d'Angleterre (1904); } \\
\text { Agrand. Hôtel de la Paix } \\
\text { (1908); transf. Hôtel } \\
\text { Beau-Rivage (1908) }\end{array}$ & \\
\hline $\begin{array}{l}\text { Quillet, Edmond } \\
(1869-1934)\end{array}$ & VE & $\begin{array}{l}\text { Transf. de } \\
\text { l'Hôtel d'Angleterre } \\
\text { (1903) }\end{array}$ & \\
\hline $\begin{array}{l}\text { Reverdin, Adolphe } \\
(1809-1901)\end{array}$ & GE & $\begin{array}{l}\text { Hôtel de l'Ecu de } \\
\text { Genève (1839-1841) }\end{array}$ & $\begin{array}{l}\text { Société de l'Hôtel de } \\
\text { l'Ecu de Genève }\end{array}$ \\
\hline $\begin{array}{l}\text { Reverdin, Emile } \\
(1845-1901)\end{array}$ & GE & & $\begin{array}{l}\text { Société des Hôtels garnis } \\
\text { de Genève }\end{array}$ \\
\hline $\begin{array}{l}\text { Rouge, Georges } \\
(1834-1920)\end{array}$ & VI & $\begin{array}{l}\text { Transf. Hôtel Byron } \\
(1899)\end{array}$ & \\
\hline $\begin{array}{l}\text { Schobinger, Henri } \\
(1857-1917)\end{array}$ & $\mathrm{VE}$ & $\begin{array}{l}\text { Agrandissement du } \\
\text { Grand Hôtel de Vevey } \\
(1898)\end{array}$ & \\
\hline $\begin{array}{l}\text { Schorp, Alphonse } \\
(1881-1949)\end{array}$ & $\mathrm{MO}$ & $\begin{array}{l}\text { Hôtel des Alpes } \\
\text { vaudoises à Glion }\end{array}$ & \\
\hline $\begin{array}{l}\text { Verrey, Jules } \\
(1822-1896)\end{array}$ & LS & & $\begin{array}{l}\text { Société immobilière } \\
\text { d'Ouchy (action.) }\end{array}$ \\
\hline
\end{tabular}




\begin{tabular}{|c|c|c|c|}
\hline Nom de l'architecte & Pôle(s) & $\begin{array}{l}\text { Réalisation(s) hôtelière(s) } \\
\text { dans l'Arc lémanique }\end{array}$ & $\begin{array}{l}\text { Administrateur et/ou } \\
\text { actionnaire dans une } \\
\text { (des) société(s) d'hôtel(s) }\end{array}$ \\
\hline $\begin{array}{l}\text { Villard, Louis (1856- } \\
\text { 1937) }\end{array}$ & $\mathrm{MO}$ & $\begin{array}{l}\text { Agrand. Hôtel } \\
\text { Excelsior et Bon-Port } \\
\text { (1893 et 1906); Hôtel de } \\
\text { Jaman (1898); Agrand. } \\
\text { Grand Hôtel des Avants } \\
\text { (surélév. 1900 et agrand. } \\
\text { 1911-1912); Hôtel } \\
\text { Splendid (1902-1904); } \\
\text { Hôtel Beau-Site à Baugy } \\
\text { (1903 et exhaus. 1913); } \\
\text { Hôtel du Parc à Glion } \\
\text { (1904-1905); Agrand. } \\
\text { Hôtel Eden (1905- } \\
\text { 1907); Transf. de l'Hôtel } \\
\text { Victoria à Glion (1907); } \\
\text { Hôtel Suisse (1905-1907 } \\
\text { et transf. 1914); Hôtel } \\
\text { de Sonloup (1911-1912) }\end{array}$ & \\
\hline $\begin{array}{l}\text { Volkart, Charles } \\
(1862-1918)\end{array}$ & MO & $\begin{array}{l}\text { Grand Hôtel des } \\
\text { Narcisses à Chamby } \\
\text { (1906); Agrand. Hôtel- } \\
\text { Pension Moser à Clarens } \\
\text { (1903- 1905); Hôtel- } \\
\text { Pension Nuss à Corseaux } \\
(1909)\end{array}$ & $\begin{array}{l}\text { Société du Grand Hôtel } \\
\text { des Narcisses et Buffet } \\
\text { Terminus de Chamby sur } \\
\text { Montreux }\end{array}$ \\
\hline
\end{tabular}




\section{BIBLIOGRAPHIE}

\section{Sources non publiées}

Archives cantonales vaudoises (ACV) : S 125/836bis-838, Onglets des actes constitutifs des sociétés anonymes de Lausanne.

Archives communales de Montreux (AM) : PP 152, Fonds de la Société immobilière de Caux.

Archives communales de Vevey (ACVevey): 95.01, Correspondance d'hôtels.

Archives d'Etat de Genève (AEG) : Jur. civ. cc. m. n. 1 à 33, Onglets des actes constitutifs des sociétés anonymes de Genève.

Archives de l'Office cantonal du registre du commerce à Moudon: Feuille officielle suisse du commerce (FOSC), 1883-1914.

Archives privées du Beau-Rivage Palace à Ouchy.

\section{Sources publiées}

Bulletin technique de la Suisse romande

Feuille d'Avis de Vevey

Gazette de Lausanne

Journal de Genève

Monneron, Robert, Les valeurs de la Bourse de Lausanne, Lausanne, Payot, 1913.

Zentralbureau des Schweizer Hotelier-Vereins (Hrsg.), Zur Erinnerung an die schweizerische Landesaustellung, Basel, 1915. 


\section{Etudes}

Breuillaud-Sottas, Françoise, La prodigieuse ascension des eaux d'Evian (1790-1914), Lille, Atelier national de reproduction des thèses, 2003.

Dupont, Patricia et Frey, Sabine, "Un paradis encadré». La fonction du tourisme à Vevey et Montreux 1880-1914, [s.1.], [s.n.], 1989.

Encyclopédie illustrée du Pays de Vaud, 3, Les artisans de la prospérité, Lausanne, 24 Heures, 1972.

FlüCKiger-Seiler, Roland, Hotelträume. Zwischen Gletschern und Palmen. Schweizer Tourismus und Hotelbau 1830-1920, Baden, Hier + Jetzt, 2001.

Lapointe, Julie, Essor et déclin de la Société immobilière de Caux (18981930). Regard sur l'histoire touristique d'une station d'altitude, Université de Lausanne, mémoire de licence, 2008 (non publié).

LÜthI, Dave, "Lavaux-Palace. L'invention d'une région touristique - 1860-1920", Revue historique vaudoise, 114 (2006), p. 181-193.

Senarclens, Jean de, van Berchem, Nathalie et Marquis, Jean M., L'hôtellerie genevoise, Genève, Société des hôteliers de Genève, 1993.

Senn, Henri-Georges, La Suisse et le tourisme, Lausanne, Payot, 1918.

Tissot, Laurent, "Hôtels, pensions, pensionnats et cliniques: fondements pour une histoire de "l'industrie des étrangers" à Lausanne, 1850-1920", in Le passé du présent. Mélanges offerts à André Lasserre, éds Brigitte Studer et Laurent Tissot, Lausanne, Payot, 1999, p. 69-88.

\section{Crédit iconographique}

Fig. 2:

Musée historique de Lausanne. 